\title{
ИСТОЧНИКИ МЕДИИРОВАНИЯ ИНВЕСТИЦИЙ В ВОЗОБНОВЛЯЕМЫЕ ИСТОЧНИКИ ЭНЕРГИИ
}

\section{(c) 2020 Конников Евгений Александрович}

кандидат экономических наук, Высшая инженерно-экономическая школа Санкт-Петербургский политехнический университет Петра Великого (СПбПУ), Россия, Санкт-Петербург E-mail: konnikov.evgeniy@gmail.com

(C) 2020 Мансуров Руслан Дамирович

Высшая инженерно-экономическая школа

Санкт-Петербургский политехнический университет Петра Великого (СПбПУ), Россия, Санкт-Петербург

E-mail:wozen@yandex.ru

\section{(C) 2020 Михеев Павел Сергеевич}

Высшая школа управления и бизнеса

Санкт-Петербургский политехнический университет Петра Великого (СПбПУ), Россия, Санкт-Петербург

E-mail: miheev.ps@edu.spbstu.ru

(c) 2020 Ниемб Бекуме Сюзанн

Высшая инженерно-экономическая школа

Санкт-Петербургский политехнический университет Петра Великого (СПбПУ), Россия, Санкт-Петербург

E-mail: nyembsuze@yahoo.fr

В условиях цифровизации экономики и расширения мировых рынков промышленной продукции продолжает неуклонно расти потребность в стабильной и экономически доступной энергии. При этом превалирующее использование исчерпаемых источников энергии провоцирует потенциальное увеличение стоимости единицы энергии в отдельных странах. Более того, усиливающееся внимание моровой общественности к вопросам устойчивого развития, провоцирует необходимость снижения экологических последствий выработки энергии, что неизбежно влияет на стоимость. В следствии данных тенденций все большее внимание привлекают возобновляемые источники энергии, потенциально являющиеся наиболее доступными и экологичными. Однако, данный рынок является достаточно волотильным, что мешает медиированию инвестиционного потока. Целью данной работы является выявление факторов, влияющих на инвестиции в возобновляемую энергетику и предложение методов воздействия, способствующих росту инвестиций. По результатам исследования была получена модель медиирования инвестиций в возобновляемые источники энергии, а также сформулированы выводы относительно природы развития данного рынка.

Ключевые слова: возобновляемые источники энергии, солнечная энергия, ветровая генерация, инвестиции.

Одной из важнейших проблем современности является антропогенное загрязнение окружающей среды. Развитие промышленности, широкое использование горючих полезных ископаемых в качестве источников энергии приводит к накоплению в атмосфере парниковых газов. Данный процесс способствует развитию другой глобальной проблемы: изменению климата. Парижское соглашение по климату, которое подписали 175 стран, является свидетельством того, что подавляющее большинство государств мира осознают масштаб проблемы и готовы приложить усилия в целях ограничения глобального роста температуры до $1,5^{\circ} \mathrm{C}$. Одним из средств достижения целей Парижского соглашения является приведение финансовых потоков в направлении развития, характеризующегося низким уровнем выбросов. Одним из 
способов снизить уровень выбросов является переход на энергию, получаемую из возобновляемых источников. С развитием технологий такая энергия приобретает все большее применение, становясь доступнее для широкого круга потребителей, однако темпов роста потребления энергии из ВИЭ по-прежнему недостаточно для достижения целей, установленных Парижским соглашением. Инвестиции в возобновляемую энергетику, будучи одним из важнейших драйверов развития этого рынка, показывают неутешительную динамику в последние годы (рисунок 1). Так, темп прироста в 2016 году составил $-8 \%$, а в 2018 году - 11\%. При сохранении подобных тенденций достижение глобальных целей ставится под угрозу.

В связи с этим целью данного исследования является выявление факторов, влияющих на инвестиции в возобновляемую энергетику и предложение методов воздействия, способствующих росту инвестиций.

Анализ теоретического базиса показал, что уровень инвестиций в возобновляемую энергетику является комплексным показателем, зависящим от множества факторов, как количественных, так и качественных. Литературный обзор позволил выявить ряд потенциально оказывающих влияние на инвестиции количественных переменных, которые можно достоверно трактовать при исследовании зависимостей на глобальном уровне. Рассмотренные труды помогли прийти к выводу, что необходимо фокусироваться на энергии, вырабатываемой при помощи солнца и ветра, поскольку именно эти два вида ВИЭ, имея невысокую себестоимость, привлекают подавляющее большинство инвестиций данной отрасли. Хочется отметить, что несмотря на весомое влияние, оказываемое государственной политикой, поддержкой в виде субсидий и льгот, мы сочли нецелесообразным рассмотрение таких факторов по ряду причин. Во-первых, достоверная количественная оценка данных факторов затруднительна. Во-вторых, интерпретация данных показателей на глобальном уровне может оказаться ошибочной ввиду того, что политики, проводимые разными странами для поддержки выработки энергии из ВИЭ, отличаются. В-третьих, намечается тенденция к переходу от мер государственного регулирования ВИЭ к рыночным инструментам стимулирования их развития. По нашему мнению, такой тренд позволяет отказаться от исследования связей между уровнем инвестиций в ВИЭ и государственной политикой и сфокусироваться на рыночных драйверах роста инвестиций в данной сфере.

В исследование включены данные с 2004 по 2016 год. Новые инвестиции в возобновляемую энергию (далее «Инвестиции») являются предметом проводимого исследования, следовательно, данный показатель будет выступать в качестве эндогенной переменной. Дополнительными направлениями исследования являются изучение влияния инвестиций на выработку электроэнергии из возобновляемых источников (далее «Выработка») и на долю потребления энергии, выработанной из возобновляемых источников, в общем объеме потребления (далее «Доля потребления»). Исследование данных связей позволит нам сделать вывод о том, можно ли влиять на Выработку и Долю потребления, изменяя уровень Инвестиций. Выработка и Доля потребления также выступают в роли эндогенных переменных. Для того чтобы установить, какова доля инвестиций в движении всех денежных средств в сфере ВИЭ мы ввели показатель «Совокупные транзакции (оборот)» (далее «Совокупные транзакции») (total transactions). Мы считаем целесо-

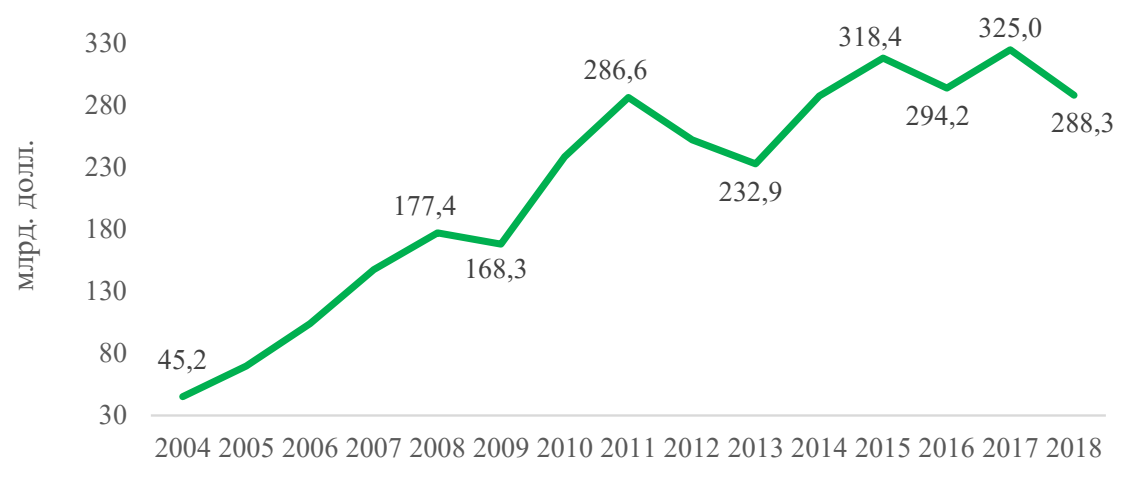

Puc. 1. Динамика инвестиций в возобновляемую энергию в мире 
образным изучение того, как уровень развитие технологий сказывается на объеме инвестиций в ВИЭ. В результате НИОКР (R\&D) создаются более эффективные и дешевые в производстве компоненты для станций ВИЭ, что повышает их конкурентоспособность. Мы предполагаем, что, это может способствовать росту инвестиций в ВИЭ. Для оценки уровня научного прогресса мы вводим переменную «Количество поданных патентов в сфере возобновляемой энергии» (далее «Количество патентов»). Отметим, что существует лаг между датой подачи патента и его официальной публикаций и появлением в базе данных PATSTAT. По этой причине данные за последние три года (2017, 2018 и 2019) не учитываются в данном исследовании. Статистика поисковых запросов Google trends позволит оценить популярность энергии из возобновляемых источников и наличие интереса к данной теме. Мы решили рассмотреть число запросов по всему миру на следующие темы: «Renewable energy», «Solar panel», «Onshore wind» и «Small turbine». Решение о включении факторов в исследование возникло в связи с тем, что по данным Nasdaq Global Information Services (статья «Clean energy \& Smart Grid Infrastructure») в последнее время отмечается рост спроса на «чистую энергию» как в корпоративном мире, так и среди домашних хозяйств. Следующими переменными, которые мы посчитали нужным включить в данное исследование, являются показатели, отражающие совокупную мощность установленных солнечных и ветряных генераторов энергии. Мы решили сфокусироваться на этих двух типах ВИЭ, по трем причинам: 1) низкая себестоимость получаемой электроэнергии; 2) ввиду этого широкая популярность и распространенность (солнечные панели лидируют по объему инвестиций среди всех остальных типов ВИЭ); 3) в результате роста эффективности солнечных или ветряных установок, требуется меньший уровень инвестиций для запуска того же уровня проектов, что повышает доступность технологий ВИЭ, а значит и инвестиционную привлекательность. Отобранные нами переменные: «Мощность солнечных элементов», подразумевающая совокупную мощность установленных солнечных панелей (solar PV) и гелиоконцентраторов (concentrated solar power), «Мощность солнечных панелей» (solar PV) отдельно, как наиболее распространенный способ преобразования солнечной энергии. «Мощность ветряных генераторов», подразумевающая со- вокупную мощность установленных наземных или еще onshore wind и прибрежных (морских) или offshore wind ветрогенераторов. Предполагая что себестоимость вырабатываемой из ВИЭ энергии является одним из значимых факторов при принятии решений об инвестировании, мы решили рассмотреть и включить в исследование такие факторы как стоимость модуля солнечной панели, общая стоимость установки наземного ветрогенератора (далее «Н.В. Общая стоимость установки», LCOE наземных ветрогенераторов (нормированная стоимость энергии, выработанной наземным ветрогенератором) (далее «Н.В. LCOE»), доля мощности наземных ветрогенераторов, используемая от потенциально доступной мощности (далее «Н.В.Доля мощности»), общая стоимость установки прибрежного (морского) ветрогенератора (далее «П.В. Общая стоимость»), LCOE прибрежных (морских) ветрогенераторов (нормированная стоимость энергии, выработанной прибрежным (морским) ветрогенератором) (далее «H.B. LCOE»), доля мощности наземных ветрогенераторов, используемая от потенциально доступной мощности (далее «Н.В.Доля от потенциальной мощности»). Мы также посчитали нужным учесть такой фактор как размер (мощность) турбины прибрежного (морского) ветрогенератора (далее «П.В.Размер турбины»). По нашему мнению, ухудшение качества воздуха способствует повышению внимания к проблемам загрязнения атмосферы и глобального потепления. По этой причине мы решили рассмотреть объем выбросов углекислого газа в атмосферу (далее «Выбросы CO_2») как один факторов, влияющих на уровень инвестиций в возобновляемую энергетику. Итоговый комплекс переменных представлен в таблице 1.

Структура связей между данными факторами может быть представлена в виде концептуальной модели (рисунок 2).

Мы предполагаем, что все включенные в исследование экзогенные переменные оказывают влияние на Инвестиции. Инвестиции, в свою очередь, влияют на Выработку и Долю потребления, поскольку являются необходимым ресурсом для развития технологий и распространения возобновляемой энергетики. Мощность солнечных элементов, солнечных панелей и ветряных генераторов помимо влияния на Инвестиции сказывается и на Выработке с Долей потребления, поскольку данные показатели характеризуют эффективность генераторов электричества и 
Таблица 1. Эндогенные и экзогенные переменные.

\begin{tabular}{|c|c|c|c|c|}
\hline № & Переменная & Тип переменной & $\begin{array}{c}\text { Условное } \\
\text { обозначение }\end{array}$ & $\begin{array}{c}\text { Единицы } \\
\text { измерения }\end{array}$ \\
\hline 1. & Инвестиции & Эндогенная & $Y$ & млрд. долл. \\
\hline 2. & Совокупные транзакции & Экзогенная & $X_{1}$ & млрд. долл. \\
\hline 3. & Количество патентов & Экзогенная & $X_{2}$ & штук \\
\hline 4. & Google trends. Renewable energy & Экзогенная & $X_{3}$ & штук \\
\hline 5. & Google trends. Solar Panel & Экзогенная & $X_{4}$ & штук \\
\hline 6. & Google trends. Onshore wind & Экзогенная & $X_{5}$ & штук \\
\hline 7. & Google trends. Small wind turbine & Экзогенная & $X_{6}$ & штук \\
\hline 8. & Выработка & Эндогенная & $Z$ & TBT·ч \\
\hline 9. & Мощность солнечных элементов & Экзогенная & $X_{7}$ & $\mathrm{MBT}$ \\
\hline 10. & Стоимость модуля солн. панели & Экзогенная & $X_{8}$ & USD/кBT \\
\hline 11. & Мощность солнечной панели & Экзогенная & $X_{9}$ & MW \\
\hline 12. & Мощность ветряных генераторов & Экзогенная & $X_{10}$ & MW \\
\hline 13. & Доля потребления & Эндогенная & $Q$ & $\%$ \\
\hline 14. & Н.В. Общая стоимость установки & Экзогенная & $X_{11}$ & USD/КBT \\
\hline 15. & H.B. LCOE & Экзогенная & $X_{12}$ & USD/кBт \\
\hline 16. & Н.В. Доля от мощности & Экзогенная & $X_{13}$ & $\%$ \\
\hline 17. & П.В. Общая стоимость установки & Экзогенная & $X_{14}$ & USD/кBт \\
\hline 18. & П.B. LCOE & Экзогенная & $X_{15}$ & $\mathrm{USD} / \mathrm{kBT}$ \\
\hline 19. & П.В. Доля от мощности & Экзогенная & $X_{16}$ & $\%$ \\
\hline 20. & П.В. Размер турбины & Экзогенная & $X_{17}$ & $\mathrm{MBT}$ \\
\hline 21. & Выбросы & Экзогенная & $X_{18}$ & млн. тонн \\
\hline
\end{tabular}

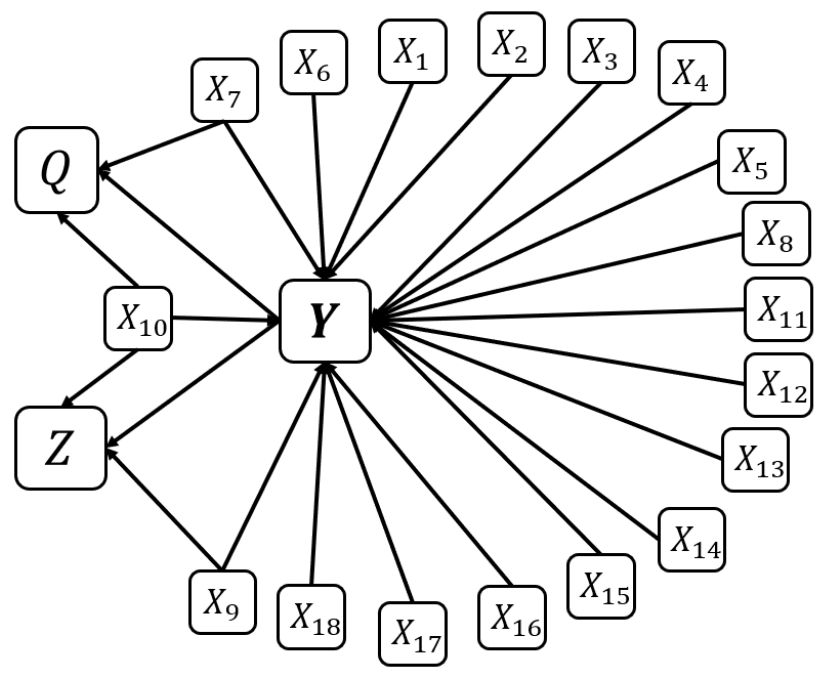

Puc. 2. Теоретическая концептуальная модель 
их потенциальную способность удовлетворить спрос на энергию.

По результатам обработки статистических данных была получена следующая модель:

$Y=422,7496+0,0055 X_{2}+0,0012 X_{7}+0,0601 X_{8}-$ $0.2901 X_{11}-382,4192 X_{16}$

Где:

1. $Y$ - инвестиции (млрд. долл.),

2. $X_{2}-$ количество патентов,

3. $X_{7}-$ мощность солнечных элементов (МBт),

4. $X_{8}-$ стоимость модуля солнечной панели (USD/кBт),

5. $X_{11}-$ Н.В. общая стоимость установки (USD/кBT),

6. $X_{16}-$ П.В. доля от мощности (\%).

Данная модель позволяет описать 98\% дисперсии (нормированный $R^{2}=98,09 \%$ ), при этом, в среднем расчетные значения отклоняются от фактических на 6\% (ошибка аппроксимации 6,17\%), что является допустимым в подобных регрессионных моделях. Полученная модель значима, поскольку при уровне доверия к модели в 90\%, заданном в методологии, F-значимость составляет $1,10 *$ Е-06. Р-уровень каждого из регрессоров также не превышает 6\%, что соответствует заданному уровню доверия к модели (при нем p-level не должен превышать 10\%). Данные характеристики свидетельствуют о том, что построенная модель с необходимой точностью описывает связи, возникающие между регрессорами и зависимой переменной.

В соответствии с нашей методологией для получения конечной формы модели из нее был исключен ряд факторов. Среди них статистика поисковых запросов в Google Trends, H.B. LCOE, П.B, LCOE, П.В. размер, Совокупные транзакции, П.В. Общая стоимость установки, Н.В. Доля от мощности, Выбросы $\mathrm{CO}_{2}$.

Динамика фактического и расчетного уровня инвестиций приведена на графике (рисунок $3)$.

В результате эвристического анализа данных показателей не было выявлено структурных выбросов. Обнаруженные структурные разрывы выделены на графике. Мы считаем, что причиной принципиального расхождения в трендах фактических и расчетных значений в 2008 и 2009 гг. послужила мировой экономическая нестабильность, вызванная экономическим кризисом. Замедление роста мировой экономики было обусловлено снижением общего уровня инвестиций, в том числе и в возобновляемую энергию. Так, в 2009 году уровень инвестиций в возобновляемую энергию снизился на 5\% со 177,4 млрд. долл. в 2008 году до 168,3 млрд. долл. в 2009. Учитывая показатели качества построенной модели и, соответственно, высокую приближенность расчетных значений к фактическим, можно сделать вывод о том, что модель значима.

Для исследования связи между Долей потребления (в качестве эндогенной переменной) и Инвестициями (в качестве экзогенной переменной) было построено две модели. Первая модель - модель парной регрессии. При том что модель получилась значимой, Инвестиции описали лишь $47 \%$ дисперсии. Вторая модель - модель множественной регрессии, в которую помимо Инвестиций в качестве экзо-

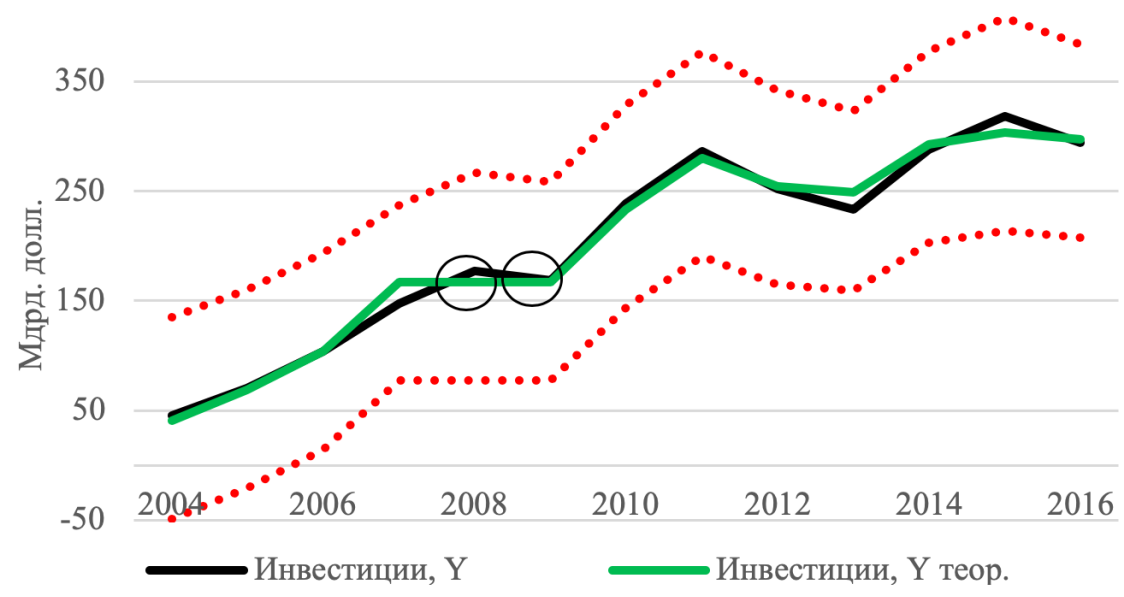

Рис. 3. Интервальный график фактических и теоретических значений 
генных переменных были добавлены факторы из таблицы 1 . В соответствии с нашей методологией из модели были исключены незначимые регрессоры. В результате, данная модель значима и описывает $98 \%$ дисперсии. Стоит отметить, что среди регрессоров отсутствуют Инвестиции. Из этого следует следующее: несмотря на то, что инвестиции, если рассматривать их отдельно, оказывают определенное влияние на Долю потребления, остальные факторы более эффективно описывают дисперсию (это подтверждается второй моделью), а значит, влиянием Инвестиций на Долю потребления можно пренебречь.

Аналогично были выявлены факторы, влияющие на Выработку. Модель парной регрессии Выработки и Инвестиций оказалась значимой, при этом описывала 75\% дисперсии, что несколько ниже установленного в рамках нашего исследования удовлетворительного уровня. В свою очередь, из значимой модели множественной регрессии, изначально включавшей в себя факторы из таблицы 1 , а в итоговой итерации, объясняющей 99\% дисперсии, Инвестиции были исключены. Получается, что, как и в случае с Долей потребления, существуют другие факторы, описывающие дисперсию эффективнее, чем Инвестиции, поэтому связь Выработки и Инвестиций несущественна.

В результате была построена новая концептуальная модель (рисунок 4), отражающая связи, выявленные в результате построения регрессионных моделей.

Наибольшее влияние на объем инвестиций в возобновляемую энергию оказывают общая стоимость установки наземных ветрогенераторов и количество поданных патентов. При этом увеличение числа поданных патентов приводит к значительному росту инвестиций, а наиболее эффективным драйвером роста вложений в возобновляемую энергию является снижение общей стоимости установки наземных ветрогенераторов электричества. Так, при увеличении количества поданных патентов на $1 \%$ от средней величины и при одновременном сокращении общей стоимости установки ветряных генераторов на $1 \%$ от средней величины, рост объема инвестиций составит в среднем 3,17\%. Следовательно, наиболее рациональными шагами для увеличения уровня Инвестиций являются стимулирование роста количества патентов и способствование созданию условий, при которых общая стоимость установки наземных ветрогенераторов будет снижаться.

Количество поданных патентов напрямую зависит от востребованности технологий возобновляемой энергии и от грантов (финансирования), выделяемых на развитие данной сферы. Важно обозначать значимость «зеленой энергии» с точки зрения глобальных целей человечества и прямые выгоды от использования энергии из возобновляемых источников для компаний и домашних домохозяйств. Это позволит создать спрос на энергию из ВИЭ, что, в свою очередь, позволит привлечь больший объем финансирования, который не только станет стимулом для ученых, но и обеспечит возможность разрабатывать более технологичные и энергоэффективные решения.

С нашей токи зрения, научные исследования и разработки позволяют сделать выработку электроэнергии из ВИЭ дешевле. Совершенствование технологий в сфере ветряной энерге-

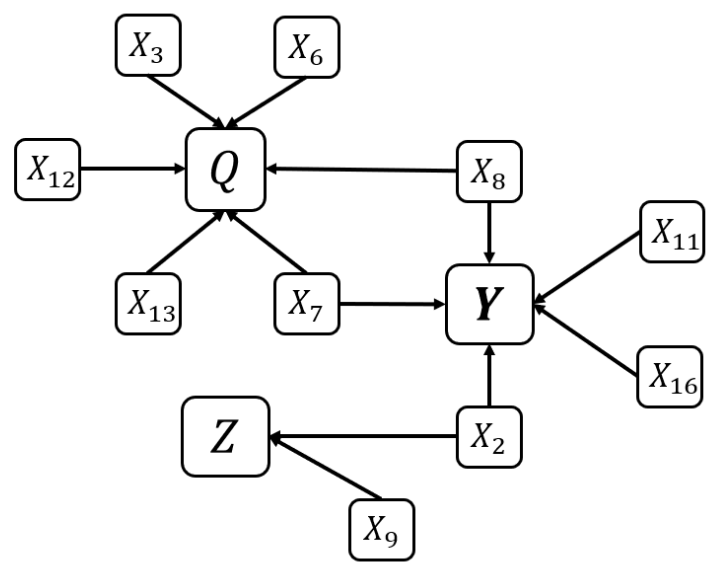

Puc. 4. Фактическая концептуальная модель 
тики будет способствовать снижению общей стоимости наземных ветрогенераторов. Кроме того, рост востребованности «зеленой энергии» позволит увеличить масштабы производства наземных ветряных генераторов что позволит экономить на масштабе с большей эффективностью (удешевление производства).

Анализируя пути увеличения Инвестиций, мы пришли к следующему выводу: рынок возобновляемой энергии противоречив. Главные драйверы роста Инвестиций, обозначенные ранее, требуют значительного объема финансирования: НИОКР (и патенты, как их результат) предоставляют новые технологии, повышающие эффективность добычи энергии и снижающие стоимость получения такой энергии за счет удешевления стоимости установок по выработке энергии. Финансирование научных разработок рождает новые технологии, повышающие инвестиционную привлекательность «зеленой энергии». Получается, что для роста Инвестиций необходимо инвестировать в возобновляемую энергетику. Мы считаем это диалектикой рынка возобновляемых источников энергии.

На первый взгляд, образуется некая замкнутость системы, когда развитие целевого показателя (Инвестиций) зависит от него самого же. Тем не менее, ситуация не является безвы- ходной. Кажущаяся замкнутость по факту не является таковой, иначе прогресс на рынке возобновляемой энергетики попросту бы отсутствовал. Переход на качественно новый уровень возможен главным образом за счет того, что развитие возобновляемой энергетики и рост Инвестиций описывают не движение по «окружности», а скорее восхождение по «спирали». И с каждым разом попадающие на рынок возобновляемой энергетики Инвестиции создают все больший потенциал для развития, а значит и для дальнейшего привлечения Инвестиций.

Инвестиции - ограниченный ресурс и одновременно локомотив рынка «зеленой энергии». Учитывая диалектику данного рынка, мы также видим возможность для увеличения Инвестиций в росте востребованности энергии из возобновляемых источников. Одним из главных мотивов инвестирования в данную сферу должно стать осознанное желание внести свой вклад в решение глобальных проблем и снизить уровень негативного влияния человека на окружающую среду. С нашей точки зрения, стимулирование осознанного спроса на «зеленую энергию» позволит придать больший импульс в развитии данной отрасли, в росте Инвестиций и обеспечит выход на новый уровень в более короткие сроки.

\section{Библиографический список}

1. .И. Бушукина. Финансирование инвестиционных проектов в области возобновляемой энергетики / В. И. Бушукина // Вестник ЮУрГУ. Серия «Энергетика».- 2019.- Т. 19, № 1.- С. 50-57. DOI: 10.14529/power190106

2. В.И. Бушукина. Развитие инвестиционных проектов в области возобновляемой энергетики (на примере оффшорных станций Европы). Сборник статей по материалам III Всероссийской (национальной) научнопрактической конференции с международным участием. Под общей редакцией С. Ф. Сухановой. 2019. С. $150-154$

3. В.С. Ахмедов. Мировой опыт инвестирования в альтернативную энергетику // Томский государственный архитектурно-строительный университет (ТГАСУ). 2018.

4. Г.С. Чеботарева, В. Стриелковски, В. А. Благинин. Рынок возобновляемой энергетики: развитие и доходность компаний // Управленец. 2019. Т. 10. № 3. C. 58-69. DOI: 10.29141/2218-5003-2019-10-3-6.

5. Т.Н. Седаш. Возобновляемые источники энергии: стимулирование инвестиций в России и за рубежом // Российский внешнеэкономический вестник. 2016. № 5. С. 50-56.

6. BP. Statistical Review of World Energy - all data 1965-2018 // BP. 2019. URL: https://www.bp.com/content/dam/ bp/business-sites/en/global/corporate/xlsx/energy-economics/statistical-review/bp-stats-review-2019-all-data. xlsx (дата обращения 10.11.2019)

7. Bindzi Zogo Emmanuel Cedrick, Wei Long. Investment Motivation in Renewable Energy: A PPP Approach // Energy Procedia. Volume 115. June 2017. Pages 229-238. DOI: 10.1016/j.egypro.2017.05.021

8. Global trends in Renewable Energy Investment 2019// Frankfurt School-UNEP Centre/BNEF. 2019

9. Google Trends. Available at: https:/trends.google.com/trends (дата обращения 08.12.2019)

10. International Renewable Energy Agency (IRENA). Decentralized Renewable Energy Data Review. Available at: http://resourceirena.irena.org/gateway/dashboard/ (дата обращения 16.11.2019)

11. Solar PV module prices vs. cumulative capacity 1976 to 2016. Our World in Data. Available at: https://ourworldindata. org/renewable-energy (дата обращения 01.12.2019) 
12. Renewables 2019 Global Status Report // REN21. 2019.

13. Ron Pernick, Efram Slen. Clean Energy \& Smart Grid Infrastructure. // Nasdaq Global Information Services. 2018.

14. The World Bank. World Development Indicators. Available at: http://databank.worldbank.org/data/home.aspx (дата обращения 20.11.2019)

15. Yongping Zhai, Yoonah Lee. Investment in renewable energy is slowing down // World Economic Forum in collaboration with Asian Development Bank. 2019. URl: https://www.weforum.org/agenda/2019/09/globalrenewable-energy-investment-slowing-down-worry/ (дата обращения 24.10.2019) 